\title{
QUANTITATIVE METHODS IN THE STUDY OF PULMONARY PATHOLOGY
}

\author{
BY \\ M. S. DUNNILL* \\ From the Department of Medicine, Columbia University, and the Cardiopulmonary Laboratory, \\ Bellevue Hospital, New York
}

Clinico-pathological correlation has always been one of the main aims of the morbid anatomist. However, although clinical physiologists have for many years expressed their results in a quantitative form, pathologists have progressed mainly, though not entirely, along a descriptive path. Descriptive pathology has reached a high degree of refinement with the study of ultra-structure by the electron microscope and the probing of cellular chemistry by histochemical methods. Little interest has been shown in the field of quantitative morpho$\operatorname{logy}$, although a quantitative study of gross and microscopic pathology, correlated with the findings of physiopathologists, might well yield much useful information. Thompson (1917) pointed out many applications of this approach to general biology, and recently Grant (1961) has drawn attention to the importance of this type of study in cardiac pathology and has referred to the work of Linzbach (1960). This paper is concerned with the methods of quantitation as applied to the lung, but the methods mentioned are capable of adaptation to almost any organ.

Pulmonary physiology is a subject which has undergone enormous advances over the past 30 years, mainly since the introduction of cardiac catheterization as a safe clinical procedure by Cournand and Ranges (1941). The relatively slow progress of pulmonary pathology is probably due to inadequate descriptions of the various aspects of emphysema and chronic lung disease. This has been remedied largely by the work on chronic bronchitis of Reid (1954) and the description of centrilobular emphysema by Leopold and Gough (1957). What is needed now is precise information concerning volumes of normal and abnormal regions of the lung, the surface area of the airtissue interface, and the number of the various units, such as alveoli and alveolar ducts, in normal and emphysematous lungs. The purpose of this paper is to show how these measurements can be

*Present address: Department of Pathology, The Radcliffe Infirmary, Oxford. made on the gross specimens available at necropsy and on histological sections with the light microscope.

The problems involved bear a striking resemblance to those of the geologists. Chayes (1956) in a remarkable book sums up the methods available to those interested in the quantitative composition of rocks. He remarks in his preface that his object is to put " numerical flesh on the bones of definition," a goal that should be the ambition of every morbid anatomist. This work requires the abandonment of such well-worn terms as "extensive fibrosis" and "a mild degree of emphysema" and their replacement by numerical values.

\section{Preparation of Organs for Quantitative ANALYSIS}

The traditional methods of performing a necropsy are often unsuited to the quantitative approach which in general requires that organs should be fixed before they are cut. It is necessary to measure the volumes of organs in the fresh unfixed state. This means, in the case of the lung, very careful removal from the cadaver, to avoid puncturing the visceral pleura, and careful inflation to a volume comparable to that present during life. This volume can be estimated approximately from measurements of the chest wall. If lung volume studies were performed during life so much the better, but it is important in this case to allow approximately $500 \mathrm{ml}$. for the volume of tissue, as opposed to air, in each lung. The physiological measurement of total lung capacity refers only to air volume. The volume of the inflated lung can then be measured by water displacement in a tank, care being taken, by means of a suitable "corking" device, to prevent escape of air from the bronchus during the measurement. The procedures of fixation, dehydration, clearing, and embedding all alter the dimensions of an organ and thus careful measure- 
ments are needed both of the volume of the fixed organ and of the size of the final histological section to determine fixation and processing constants.

Various methods of lung fixation have been tried, usually involving infusion of fluid formalin along the trachea or main bronchi, and these have been reviewed by Heard (1960). Such methods, particularly when performed under considerable pressure, are unsuitable for quantitative studies, as has been pointed out by Hartung (1962). The procedure described by Weibel and Vidone (1961) using formalin steam is preferable to any other method. The lung can be expanded to the desired dimensions in a controlled manner by means of "negative" pressures similar to the intrapleural pressures present during life. The lung retains its shape when removed from the fixing chamber, and there is practically no lateral flattening which is so common with fluid fixation. The final histological slides have a beautifully clean appearance, and the delicate bronchial epithelium is preserved in a manner not seen with any other method. The volume of the fixed lung can be measured by water displacement with remarkably little deformity, particularly if it has been floated in Zenker's solution overnight. The volume of the fixed lung is less than the volume of the fresh organ, and the expression $\frac{\text { Volume of fresh lung }}{\text { Volume of fixed lung }}=\mathrm{f}^{3}$ will give the volume fixation constant for conversion of fixed to fresh lung. The constant for area measurements will of course be $\mathrm{f}^{2}$ and for linear measurements $f$. Similar processing constants $\left(\mathrm{p}^{3}, \mathrm{p}^{2}\right.$, and $\left.\mathrm{p}\right)$ can be obtained by measuring the size of the blocks taken from the fixed specimen and comparing these measurements with the size of the histological section on the slide. The reciprocals of these values will give the constants for converting dimensions of fresh to fixed material and fixed to processed material. It is necessary to carry out this procedure in every instance as these constants vary from case to case with the age of the individual and the disease process.

\section{ASSESSMENT OF Volumes ON THE GRoss (FIXED) SPECIMEN}

In order to estimate the volumes of normal and of diseased tissue in an organ the method employed must be sufficiently accurate, applicable to tissues of considerable complexity, and simple to use. All the methods available depend upon the principle first announced by the geologist
Delesse (1848) that in a complex mineral the areal proportions of a section of the mineral are equivalent to the volume proportions. The mathematical proof of this principle was not given until over 100 years later by Chayes (1954), although it had been in use as a tool of geologists since 1916 when Shand invented his recording micrometer. The problem thus resolves itself into the determination of areas or ratio of areas of various components on a cut surface.

Rosiwal (1898) extended the principle by proposing that " if the fraction of a line traversing a given component on a section can be determined, this linear fraction provides a good estimate of the fraction of the volume occupied by a given component." The length occupied by each component on the lines was measured, and it can be proved mathematically that the total lengths of line occupied by the various components are proportional to the areas of the components and hence to the volumes. It was to ease the tediousness of this linear measurement in geology that Shand invented his micrometer. On gross anatomical specimens the procedure would, however, be formidable, although integrating eyepieces are available which use the same principle for the analysis of histological sections. Most linear measurements use a system of straight, equidistant, parallel lines, and although this may give consistent results they are not entirely free from bias. In gross pathology camera lucida drawing and planimetry might be employed in area estimation, but in an organ as complex as the lung this would be excessively tedious.

It is, however, possible to perform the task of area ratio estimation in a manner which is both speedy and accurate, and also free from bias, by the point-counting method. It has been employed on histological sections by Chalkley (1943), and in petrographic analysis by Chayes (1956), but it does not appear to have been used in gross pathology. Chalkley approached the problem from the concept of a mathematical point moving randomly through a tissue. The proportion of its path resting on the various constituents will, if the path is infinitely prolonged, approach as a limit the proportions of the fractions of volume occupied by these constituents. Chayes (1956), on the other hand, uses the Delesse principle of the equivalence of areal and volume proportions and then gives a mathematical proof of the efficacy of point sums as estimators of relative areas. Thus, in Fig. 1, if $\mathrm{N}$ random points were placed on the whole area the ratio of the shaded area to the 
whole area would be the same as the ratio of the number of points, $n$, lying in the shaded area

to N. That is, $\frac{\text { Area of shaded part }}{\text { Total area }}=\frac{\mathrm{n}}{\mathrm{N}}$.

Obviously the more points that are assessed the greater the accuracy of the method. That it is possible to obtain a very high degree of precision has been shown by Chayes and Fairbairn (1951).

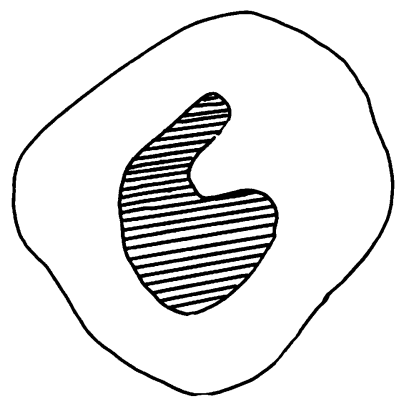

FIG. 1. The proportion of random points lying in the shaded area will be proportional to its area and still be independent of its shape.

In practice the lung is bisected through the hilum and the cut surfaces placed face downwards on a wooden board fitted with Perspex runners $1 \mathrm{~cm}$. deep. The lung is then cut into $1 \mathrm{~cm}$. thick slices using a specially made 17 in. ham knife. The slices are then laid down on a table in a good light and a piece of transparent cellophane, with the point-counting grid drawn on it, is placed over them. The points iie $1 \mathrm{~cm}$. apart and are situated at the angles of equilateral triangles of side $1 \mathrm{~cm}$. (Fig. 2). This distance of separation has been found to give good precision and reproducibility in the lung, which is the seat of pulmonary emphysema, although when assessing other organs the points may have to be placed closer together. The next step is to define the components which are to be evaluated. In pulmonary emphysema the following components have been found satisfactory: (1) Nonparenchyma (bronchi and blood vessels down to $2 \mathrm{~mm}$. diameter): this normally occupies about $10 \%$ by volume of the normal lung and rather less in emphysema; (2) normal parenchyma; and (3) abnormal air spaces. These components are then listed and, with the aid of a hand lens, the tissue lying under each point in the grid is assessed. The process is repeated for each slice. If the three components to be measured occupy fractions $P$, $Q$, and $R$ of the total of volume $V$, then the number of points $p, q$, and $r$ counted in these fractions will be in the same relation to each other as are the volumes, i.e., $\mathrm{p}: \mathrm{q}: \mathrm{r}:: \mathrm{P}: \mathrm{Q}: \mathrm{R}$. If the total volume of the fixed lung is known, then the volume occupied by the various components can be calculated both in the fixed specimen and, using the fixation constants, in the fresh specimen. It is of course important in defining the components to be measured to ensure that together they make up the total lung volume, i.e., $P+Q+R=100 \%$ $=\mathrm{V}$.

In most lungs some 2,000 to 3,000 points are assessed ; this may seem an arduous task, but with practice, and an assistant to record the findings, both lungs can be assessed within 30 to 40 minutes. The method is reproducible by separate observers to within $5 \%$. It can also be used to evaluate quantitative regional differences in the lung. It should be noted that this method assumes that, although the total volume of the lung alters with fixation, the volume proportions remain constant.

Before leaving consideration of the gross specimen, it should be pointed out that measurement of the linear dimensions of emphysematous spaces, particularly centrilobular spaces, is possible at this

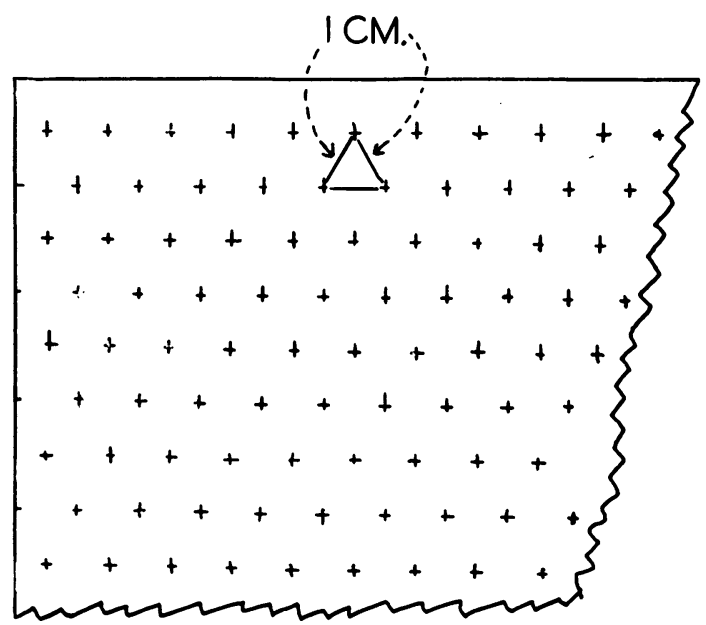

FIG. 2. A diagram to show the arrangement of points in a point-counting grid used for the estimation of volume proportions on the gross specimen. The points are arranged at the angles of equilateral triangles of side $1 \mathrm{~cm}$.

stage. A frequency distribution diagram of the size of these spaces can be constructed and also the size of the spaces related to their regional distribution. What is needed, however, is measurement of the number and surfaces of the various components (alveoli, ducts, emphysematous spaces) 
in the normal and abnormal areas, and for this analysis it is necessary to have histological material.

\section{SAMPLING OF THE LUNG}

In any histological analysis of the lung sampling is all important. In the normal organ few problems arise as, apart from a small region near the hilum, the parenchyma is fairly uniform. In a lung which is the seat of emphysema or other chronic lung disease uniformity may be present, but more often it is strikingly absent. It might be possible to examine the entire lung histologically, but the labour, time, and expense of this procedure render it expedient to select blocks of tissue of moderate dimensions to be submitted for detailed examination. It is an important premise that the selection of these samples must ensure that they give a picture of the whole organ, not just of the "interesting" areas. This represents a radical departure from the normal method of selecting tissue for histological examination. This type of sampling must of course receive somewhat hostile criticism until its efficacy is accepted. Objections were levelled at the small sample methods when they were introduced into botanical and agricultural research, though to-day they are accepted in their entirety.

Two methods are available. The first is systematic sampling, and this involves taking blocks of tissue at given intervals throughout the lung. Its main disadvantage is that, if the diseased areas have a similar type of arrangement to the sampling pattern, the sample will be unrepresentative of the lung as a whole, and any results deduced from such samples will be hopelessly wide of the true state of affairs. The alternative is random sampling, the selection of blocks by means of a random number table. In theory this method has the property of securing a small group of blocks of tissue possessing the same characteristics as the entire lung, i.e., the same proportion in which each special feature is present or absent. In practice in a totally random sample, where each of the sample units has an equal chance of selection, a highly unrepresentative selection is possible, e.g., two adjacent blocks of tissue from the upper lobe and no blocks from the lower lobe in a given slice of lung. To prevent the occurrence of this type of selection a compromise between random and systematic sampling is employed. This is the principle of stratified random sampling which retains the advantages of random sampling but imposes some restrictions on the fluctuations encountered in a simple random sample. The theory and justification of stratified random sampling have been rigorously treated and discussed by Hendricks (1956).

The method used in the lung employs grids, consisting of squares of side $1 \mathrm{~cm}$. (Fig. 3), drawn on pieces of transparent cellophane. Each square

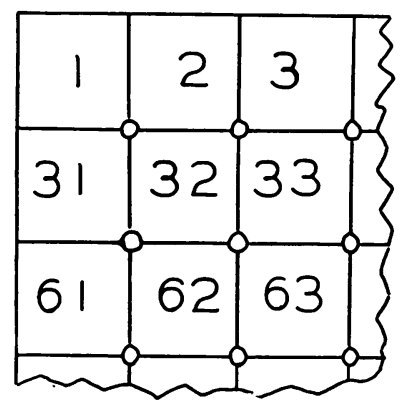

FIG. 3. A diagram to show the design of the grid used in stratified random sampling of the lung. The squares are of side $1 \mathrm{~cm}$. The perforations at the corners of each square are large enough to admit a pin head.

is numbered, and the corners of the squares are perforated. The grids are made of such a size that each will cover entirely a slice of lung. A grid is placed over each slice. If it is proposed to take 40 blocks for histological study from a pair of lungs and there are five slices for each lung, four blocks will be taken from each slice. In fact all the slices will not be of the same size, and the number of blocks taken from each slice will be determined by the size of the slice. Furthermore, employing a system of stratification means selecting the first block in each slice on a random number basis, and subsequent blocks are chosen by addition of a constant number. This avoids having two adjacent samples. The selected squares on the grid are marked with a pin which passes through the perforation at one corner into the underlying specimen. When all the blocks have been marked the grids are removed, leaving the pins in situ, and the blocks are cut out. Blocks are taken, using an arbitrary convention, with the pin situated at the top right-hand corner of the block. As near as possible blocks should be of a standard size; $2.8 \times 2 \times 1 \mathrm{~cm}$. is convenient, but whatever their size each block must be measured carefully so that it can be compared with the size of the final section on the slide to determine the processing constant referred to above. Samples are not taken from the hilar regions where large bronchi and blood vessels are present. 
Analysis of Histological Sections

Volume Proportions.-The methods available for volumetric analysis all depend on the Delesse principle which has already been discussed. There are two types of integrating eyepiece available for this work. The first employs the linear measurement principle of Rosiwal (1898) and is somewhat tedious if used for any length of time. The second depends on the point-counting principle already mentioned when dealing with the gross specimen. This was the method used by Chalkley (1943). The instrument devised by Chalkley employed only four points. This seems a very small number, but too many points in one microscopic field would be difficult to assess. Twenty-five would seem to be a reasonable number. Such eyepieces are available and are easy and rapid to operate. The points are arranged at the angles of equilateral triangles (Fig. 4).

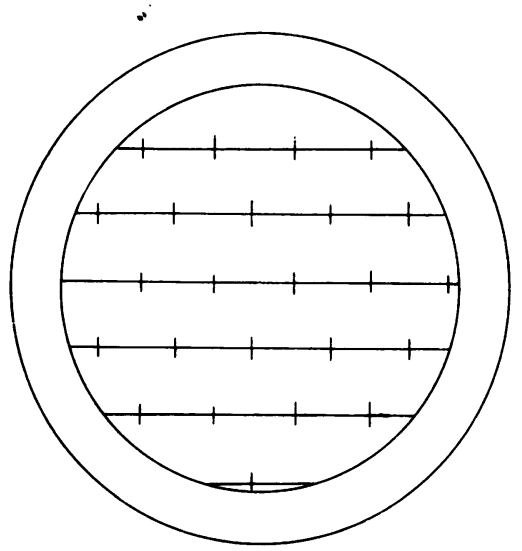

FIG. 4. A diagram of the integrating eyepiece of the point counting variety. Points are arranged at the angles of equilateral triangles. A peripheral rim of the microscopical field is left out of the grid as this is the region of greatest spherical aberration where the exact location of a point on a section is difficult to determine. This is the design of the Zeiss integrating eyepiece.

This is a much more precise procedure than that employed on the gross specimen, and furthermore different parameters are evaluated. In most cases of emphysema the following might well be assessed : alveolar air, alveolar duct air, abnormal air space, tissue, and vessels. The position of each point in the eyepiece is then recorded according to which constituent it lies in on the histological section. A hand-operated counting device, similar to those used for differential blood counts, has the advantage that results can be written down every 100 points instead of the laborious procedure of recording on paper the position of each point as it is observed. This method is simple, rapid, and independent of the shapes of the various components. The entire section can be covered with about 500 points if a low-power objective is used. The objective chosen will depend on what is finally required. Chalkley used this method to determine nuclear cytoplasmic ratios, and obviously for this work a very high power is needed. The assessment of the volume proportions in the lung requires the recording of between 500 and 1,000 points per section for consistent results. The selection of the microscopic fields is important as large bronchi and blood vessels $(>2 \mathrm{~mm}$. diameter) must be avoided, since these are classed as non-parenchyma. When the situation of the points on each slide has been observed and recorded the totals are added up. The proportions of the sums of the points in each component are then taken to equal the volume proportions of each component.

Surface Area Estimation.-One of the most important quantitative measurements to be made in the lungs is that of the internal surface area or air-tissue interface. The method of measuring this by the mean linear intercept is both simple and accurate. Its development is interesting. Tomkeieff (1945) appears to have been the first to have drawn attention to the method of finding the surface area of small objects from their area of projection. As with so many of these methods he was at first interested in the grain size of rocks. This was developed by Campbell and Tomkeieff (1952) to include a number of small bodies such as alveoli in the lung. They state that "if a number of similar bodies, each of volume $V$ and surface area $A$, are enclosed at random in a unit volume $\mathrm{V}$ and this volume is traversed by a number of lines of total length $T$, if also $L$ is the total length of these lines which is interior to the convex bodies and $\mathbf{N}$ is the number of intercepts, then

$$
\frac{\mathrm{L}}{\mathrm{T}} \longrightarrow \frac{\text { Volume of convex bodies }}{\text { Unit volume }}=\frac{\mathrm{L} \mathrm{\Sigma A}_{\mathrm{A}}}{4 \mathrm{NV}}
$$

and in the limit $\frac{\sum \mathrm{A}}{\mathrm{V}}=\frac{4 \mathrm{~N}}{\mathrm{~T}} \quad$."

Campbell and Tomkeieff (1952) also demonstrated that the bodies need not be similar to one another in shape or entirely enclosed in a unit volume. It is necessary, however, that the bodies should be randomly disposed towards the lines of 
traverse. This method was employed by Duguid, Hulse, Richardson, and Young (1953) to calculate the respiratory surface area of the lung in experimental animals.

Short (1950), in studying alveolar epithelium in relation to growth of the lung, gave a similar formulation for the calculation of the internal surface area of the lung. The proof that he offered was somewhat different and he appeared unaware of Tomkeieff's work. Hennig (1956a and $b ; 1957)$ also arrived at this formulation independently, and developed it further to include the estimation of surface area of orientated structures such as intestinal villi.

In the application of this method to the lung certain practical points must be noted. The traverses best employed for this_work are crossed hair lines, of equal and known length (Fig. 5),

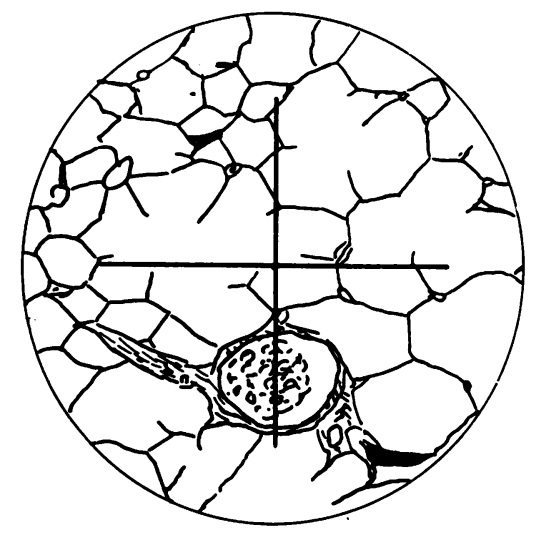

FIG. 5. A diagram of a lung field with crossed hair lines present in the eyepiece for use in the mean linear intercept method for the determination of the area of the air-tissue interface. A cut into the large vessel drawn in the lower part of the field would count as half an intercept as would a cut out of the vessel. The traverse passing through an alveolar partition is registered as a full intercept.

fitted to an eyepiece. The use of the two hair lines, at right angles to one another, compensates for any deformation that may occur to the section during cutting and mounting. The selection of microscopical fields on the section presents a problem. It is possible to count every field on any given section, but this is extremely time-consuming and some form of sampling is needed. A simple stratified random sampling system can be used, similar to that described for the selection of blocks. The micrometer gauges on the mechanical stage of the microscope are employed together with a table of six digit random numbers. The first three digits are used to give the references on the vertical scale and the second three the reference on the horizontal scale. Using this method 10 fields are easily selected. In order to avoid adjacent fields two readings can be made on the horizontal scale to every one on the vertical scale. The next point that arises is how to deal with traverses which cut structures other than alveolar walls. The situation is shown in Fig. 5 . Here a cut through an alveolar wall counts as a single intercept. A cut into a blood vessel wall, however, counts as a half, as does a cut out of a blood vessel wall. The number of intercepts is counted on both the horizontal and the vertical hair line for each field and the sum for all the sections obtained. The mean linear intercept $\mathbf{L}_{m}$ is then calculated from $m$, the sum of all the intercepts, $\mathbf{L}$, the length of the traverses, and $\mathbf{N}$, the number of times the traverses are placed on the lung, i.e., in this case twice for each field

examined, $\mathrm{L}_{\mathrm{m}}=\frac{\mathrm{N} \cdot \mathrm{L}}{\mathrm{m}}$.

The surface area of the air-tissue interface $S$ is then given by $\mathrm{S}=\frac{4 \cdot \lambda \cdot \mathrm{V} \cdot \mathrm{p}^{2} \cdot \mathrm{f}^{2} \text {. }}{\mathrm{L}_{\mathrm{m}}}$ where $\mathrm{V}=$ volume of the processed lung calculated from the fresh lung by applying the volume constants for processing and fixation; $\lambda=$ fraction of lung containing parenchyma as previously defined; $\mathrm{p}^{2}=$ constant for converting areas of processed to fixed tissue ; and $\mathrm{f}^{2}=$ constant for converting areas of fixed to fresh tissue.

This method has the great advantage that it is possible to calculate the air-tissue interface in conditions such as emphysema where the bodies are by the very nature of the disease not uniform in size. Duguid et al. (1953) used an electronic recording device for the counting of the intercepts. While this is an ingenious method it is a little difficult to see how such a method can allow for small blood vessels, and also how recording of a hit on a piece of alveolar debris can be avoided. In fact, counting by eye is both rapid and consistent.

Estimation of Numbers.-One of the serious deterrents to a quantitative approach to morbid anatomy has been the lack of a suitable method of estimating accurately the number of units in a given volume from counting the number of units in a given area on a histological slide. This problem has been very beautifully solved by Weibel and Gomez (1962). They have shown that 
the number, $\mathrm{N}$, of randomly distributed bodies in a unit volume is given by $\mathrm{N}=\frac{\mathrm{n}_{\frac{3}{2}}^{\frac{3}{2}}}{\beta \sqrt{\rho}}$ where $\mathrm{n}=$ the number of transections of the bodies on a unit area in a random section; $\rho=$ the fraction of the entire volume occupied by the bodies; and $\beta=a$ constant depending on the shape but not the size of the bodies.

The principle is only applicable to sections which are infinitely thin in comparison with the size of the bodies under investigation. In the case of alveoli, of average diameter $250 \mu$, a histological section of thickness $5 \mu$ is satisfactory. If, however, it is desired to count the number of cells in an organ such as the pituitary, a $5 \mu$ thick section would obviously be inadequate as the section would be of the same order of thickness as the diameter of the cell being counted (approximately $15 \mu$ ). Thus for cytological counts electron microscope sections would be required. An alternative might be to use optically plane objectives and count only those cells in the plane in focus. The shape constant $\beta$ has been determined mathematically by Weibel and Gomez (1962) for various bodies. The shape of a normal alveolus described by Hartroft and Macklin (1944) as a truncated cone surmounted by a cone was taken, and this gave a value of 1.55 .

An eyepiece grid is used to count the number of alveoli seen in a known area. The dimensions of the grid are measured and then the number of transections seen in the area outlined by the grid is counted. A convention is used similar to that used in counting white blood cells, i.e., alveoli cutting the top line and the left-hand line of the grid are counted as being within the area and those cutting the right-hand and bottom line are considered outside the area. On any given slide the number of alveoli are counted in 10 fields, selected by the same method of sampling as that used in the mean linear intercept method. The mean number of alveoli per field is then calculated from the counts on all the sections, the number per unit area calculated, and thus the number per unit volume deduced. As the volume of the lung parenchyma is known the total number of alveoli can be calculated. Weibel and Gomez (1962) have given a figure of $296.10^{6}$ as the number of alveoli in the normal adult lung.

This method has been used by Dunnill (1962) for the determination of the number of abnormal air spaces in the lung. A different shape constant, $\beta$, is used which, if these spaces are considered as spheres, is approximately 1.37 .
If the number of alveoli and their volume proportion in the lung are known the surface area of the alveoli can be calculated. The surface $S$ of any body will be related to its volume $\mathrm{V}$ by the equation $S=K V^{3}$ where $K$ is a constant related to the shape of the body. Weibel (1962) has found a value of 4.8 for this constant in the case of the alveolus. The mean volume of single alveolus $\mathrm{V}$ will be obtainable from the volume $\mathrm{V}$ occupied by the lung parenchyma, $\gamma$ the proportion of this volume occupied by the alveoli, and $\mathrm{N}$ the total number of alveoli by the following equation $\mathrm{V}=\frac{\gamma \cdot \mathrm{V}}{\mathrm{N}}$.

From this it follows that the total surface $S$ of the alveolar air-tissue interface will be given by $\mathrm{S}=\mathrm{NK}\left(\frac{\gamma \mathrm{V}}{\mathrm{N}}\right)^{\frac{2}{3}}$.

The implications of this are interesting as it provides a good check, in the normal lung, on the surface area calculated from the mean linear intercept method. In the emphysematous lung it is even more useful as it provides an estimate of the area of the air-tissue interface of the alveoli as opposed to the total air-tissue interface due to alveoli and emphysematous spaces, which is estimated by the mean linear intercept method.

\section{ASSESSMENT OF RESUlts}

In a normal lung the standard deviation of the mean for each of the histological measurements (mean linear intercept, alveolar transections, etc.) will be relatively small, as the lung parenchyma will be of uniform composition. This applies not only to the microscopic samples obtained on any given slide but also to the combination of means taken from each slide. The standard error of the mean, which depends on the square root of the number of the observations, can thus be reduced to a very small figure with relatively few observations. In an emphysematous lung the standard deviation between samples on any given slide, due to the nature of the disease, is bound to be large because in some fields there may be few alveoli or even none at all. Frequency distribution histograms of the intercepts on a normal and on an emphysematous lung (Fig. 6) illustrate this point.

The assessment of the standard error of the mean in these two cases will be different. In the normal lung, with its uniform distribution, the standard error, $\sigma_{\overline{\mathbf{x}}}^{-}$, may be calculated simply from the equation $\sigma_{\overline{\mathrm{x}}}=\frac{\sigma}{\sqrt{\mathrm{n}}}$ where $\sigma=$ the standard 


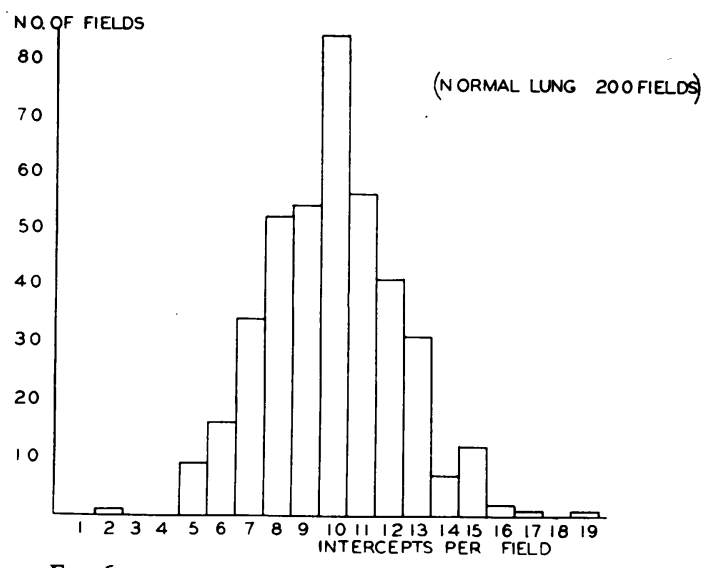

Fig. 6a

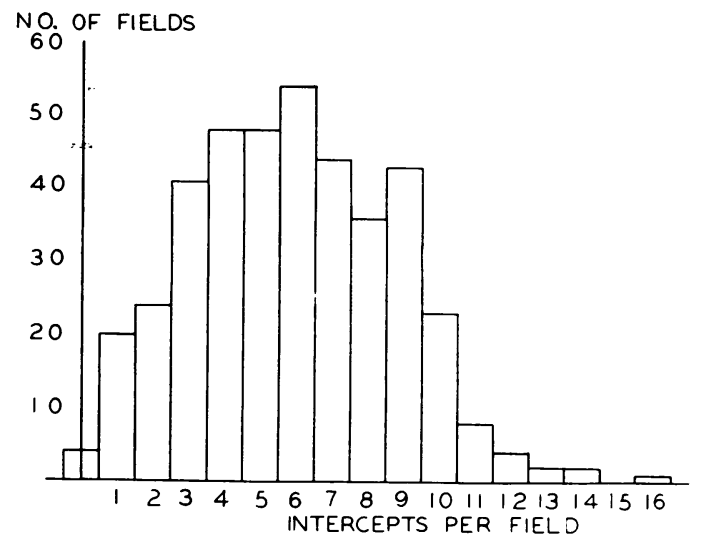

Fig. 6b

FIG. 6. Frequency distribution diagrams of intercepts in (a) a normal lung showing a relatively narrow distribution of intercepts ; and (b) an emphysematous lung; there is a much wider spread of the distribution of the intercepts.

deviation and $\mathrm{n}=$ the number of observations (i.e., number of fields counted for alveolar transections or linear intercepts).

In the pathological lung, with its wide variation between samples on the histological section and between the various sections, the following formula for finding the standard error $\sigma_{\bar{x}}$ of the mean from a stratified random sample, recommended by Arkin and Colton (1956), is used:

$\sigma_{\overline{\mathbf{x}}}=\sqrt{\frac{\sum\left(N_{s}^{2} \sigma_{\bar{x}_{s}}^{2}\right)}{N^{2}}}$ where $\sigma_{\bar{x}_{s}}=$ standard

error of the mean for each slide; $\mathrm{N}_{\mathbf{s}}=$ number of observations in each slide; and $\mathrm{N}=$ number of observations in the entire lung.

Obviously in a pathological lung many more observations will have to be made than in a normal lung, but the actual number will vary from case to case and will depend on how evenly the disease process is distributed throughout the lung. The number of blocks taken for study by stratified random sampling to obtain any given standard error cannot be predetermined, but in a lung which is the seat of $50 \%$ emphysema, as determined by the point-counting method on the gross specimen, 20 blocks of tissue will give a standard error for alveolar transections of approximately $5 \%$. This will not be the error of the estimate of the surface area of the air-tissue interface or number of alveoli, because these will depend also on the error involved in measuring the lung volume and that involved in ascertaining the volume proportions.

The estimation of the error in the determination of the volume proportions by the point-counting method, either in the gross specimens or on the histological slide, will be estimated somewhat differently. The standard error, $r$, of a percentage area will be given by $r=\sqrt{\frac{A(100-A)}{N}}$ where $A=$ the area estimated expressed as a percentage of the whole area and $\mathrm{N}=$ the total number of points counted.

It is obvious that the smaller the area to be estimated the larger the number of points that must be counted for any given error. Furthermore, it can be seen that, as the error depends on the square root of the number of observations, to decrease the error by half requires four times the number of observations. In fact the estimation of the error of the volume proportions, as determined by the point-counting integrating eyepiece, is rendered very simple because the manufacturers of these eyepieces provide a normogram which enables the error for any volume proportion to be determined. These normograms can be used to determine the number of points which must be assessed for differing proportions to be within a given range of error.

\section{CONCLUSION}

These remarks have been concerned entirely with the methods available for quantitative evaluation of pathological changes in the lung. The methods themselves are, however, adaptable to other organs and systems. An attempt has been made to analyse the available methods, most 
of which are in the geological literature and have received mathematical proof. The adaptation of instrumentation from the geological to the histological field is simple, and adequate types of integrating eyepiece are readily available. It would seem that the time is ripe for further development in the field of quantitative morphology. In certain areas of clinico-pathological correlation, notably in the lung, an abundance of this type of quantitative information is needed.

\section{SUMMARY}

Progress in the correlation of pulmonary pathology with pulmonary function studies is dependent on the collection of quantitative morphological data. This is especially important in the various forms of emphysema which have been studied extensively by sophisticated physiological techniques. Methods are described for preparing and sampling the pathological lung and for determining quantitatively (1) the volume proportions of normal and abnormal lung, (2) the surface area of the air-tissue interface of normal and abnormal lung, and (3) the numbers of normal alveoli and of abnormal air spaces in emphysema.

I am most grateful to Drs. E. R. Weibel and D. Gomez for introducing me to their counting principle before its publication. It is a pleasure to acknowledge the considerable help and encouragement received from them and from Drs. André Cournand and Dickinson W. Richards. The work was supported by grants from the Health Research Council of the City of New York, the United States Public Health Service (USPHS-H-5741 (R1)), and the New York Heart Association.

\section{REFERENCES}

Arkin, H., and Colton, R. R. (1956). Statistical Methods, 4th ed. (rev.), p. 117. Barnes and Noble, New York.

Campbell, H., and Tomkeieff, S. A. (1952). Nature (Lond.), 170, 117. Chalkley, H. W. (1943). J. nat. Cancer Inst., 4, 47.

Chayes, F. (1954). J. Geology, 62, 92.

- (1956). Petrographic Modal Analysis. Wiley, New York

and Fairbairn, H. W. (1951). American Mineralogist, 36, 704. Cournand, A., and Ranges, H. A. (1941). Proc. Soc. exp. Biol. $(N . Y), 46,462$.

Delesse, A. (1848). Annales des Mines, 13, 378.

Duguid, J. B., Hulse, E. V., Richardson, M. W., and Young, A. E. (1953). J. Physiol., 121, 8P

Dunnill, M. S. (1962). Thorax, 17, 329.

Grant, R. P. (1961). Circulation, 23, 645. Hartroft, W. S., and Macklin, C. C. (1944). Trans. roy. Soc. Can.
Sect. V, 38, 51.

Hartung, W. (1962). Amer. Rev. resp. Dis., 85, 287.

Heard, B. E. (1960). Ibid., 82, 792.

Hendricks, W. A. (1956). The Mathematical Theory of Sampling, p. 120. Scarecrow Press, New Brunswick, N.J.

Hennig, A. (1956a). Mikroskopie, 11, 1.

- (1956b). Ibid., 11, 206.

- (1957). Ibid., 12, 174.

Leopold, J. G., and Gough, J. (1957). Thorax, 12, 219.

Linzbach, A. J. (1960). Amer. J. Cardiol., 5, 370.

Reid, L. McA. (1954). Lancet, 1, 275.

Rosiwal, A. (1898). Verh. Kongr. Geol. Reichsanst. Wien, 6, 143.

Short, R. H. D. (1950). Phil. Trans. B, 235, 35.

Thompson, D'Arcy W. (1917). On Growth and Form, 1st ed. University Press, Cambridge.

Tomkeieff, S. A. (1945). Nature (Lond.), 155, 24.

Weibel, E. R. (1962). Personal communication.

— and Gomez, D. M. (1962). J. appl. Physiol., 17, 343.

- and Vidone, R. A. (1961). Amer. Rev. resp. Dis., 84, 856 